\title{
Evaluating distances using pictorial symbols in Augmented Reality Systems for indoor environments
}

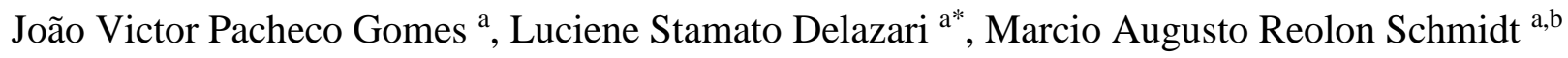 \\ ${ }^{a}$ Federal University of Paraná, Geodetic Sciences Graduate Program, Brazil, joaovictorpac@gmail.com, luciene@ufpr.br \\ ${ }^{b}$ Federal University of Uberlândia, Geodetic Sciences Graduate Program, Brazil, marcio.schmidt@gmail.com \\ * Corresponding author
}

Keywords: Augmented Reality; Indoor; Symbols; Reference Points

\begin{abstract}
:
The technology has influenced the creation and availability of indoor maps, making it possible to represent the indoor environments in different platforms such as computers and smartphones (Basiri et al., 2017 and Gao et al., 2018). The availability of indoor maps is not common, except for the You Are Here maps (Gao et al, 2018). However, mobile devices allowed new ways for map access, mainly due to the raise in its functionalities such as touch screens, internet access and GNSS signals (Nossum, 2013).
\end{abstract}

It is in this context that Augmented Reality (AR) emerges as a way of presenting geospatial information, allowing the mix of virtual information with the real world. The possibility of using smartphones as interface for AR visualization can facilitate the spatial orientation (Dick et al, 2016). Some researches that seeks to implement the AR as a technology for indoor representation highlight the importance of studies related to correct use of cartographic language (Li et al., 2019, Halik \& Medyńska-Gulij, 2016).

The research of Halik \& Medyńska-Gulij (2016), Stanek \& Friedmannova (2010) and Halik (2012) present some guidelines for design and application of symbols in the context of AR. According to these authors, in AR systems, the qualitative information should be presented through pictorial symbols disposed at the scene. For this reason and considering the interpretation for the information by the user, these authors also indicate the use of pictorial symbols for cartographic representation in AR (Halik \& Medyńska-Gulij, 2016, Stanek \& Friedmannova, 2010).

The information's decoding process from cultural knowledge is part of a broader theory known as Gestalt. The Gestalt refers to the comprehension of the object by its different attributes, mainly its shape. Studies about pictorial symbols designed based on Gestalt laws for cartographic representations in AR systems are incipient, especially in the context of mobile devices and egocentric view. The actual research refers to experiments using AR in outdoor environments and there are few experiments for the indoor space (Vanclooster et al., 2016; Nossum, 2013; Rantakari et al., 2017). Thus, it is important to investigate if pictorial symbols, designed considering the Gestalt laws and applied in indoor AR systems, could provide the correct perception of object's distance or proximity.

Considering this context, the hypothesis is that in 3D representations the notion of distance or proximity can be simulated using pictorial symbols based on the Gestalt laws; then, applying the same laws in an AR representation for an indoor environment it will be possible to perceive both depth and distance effects at same way. The hypothesis validation is accomplished through user tests where users are presented to pairs of pictorial symbols with different sizes in a simulated AR of an indoor space. The research evaluates the perceptive ability of users to estimate the proximity of reference points represented using the pictorial symbols.

The study area was a set of buildings located ate Federal University of Parana (Brazil). The structure of these buildings is complex and comprises two floors where there are several elements that can be considered as reference points, such as bathrooms, stairs, elevators, exit and access ramp. These elements were considered as reference points based on previous research conducted in the same area (Sarot, 2015 and Antunes, 2016). The pictorial symbols were designed by Sarot (2020). The symbols were changed to adapt to AR being the main alteration the removal of the white background, making the symbols transparent with figures in black (Figure 1). 


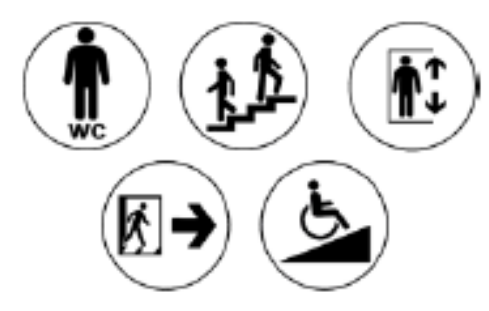

Figure 1. Pictorial Symbols used in the study (Sarot, 2020).

Figure 2 shows the scenario 1, where symbols are presented in an environment of high contrast and few shadows. The second scenario has medium contrast and natural light with some shadows. The third scenario presents low contrast, few natural light and lot of shadows. The experiment was designed for the user to identify the closest symbol, when comparing between the two symbols presented. The scenario was a simulation of an AR environment but there was no interaction allowed.

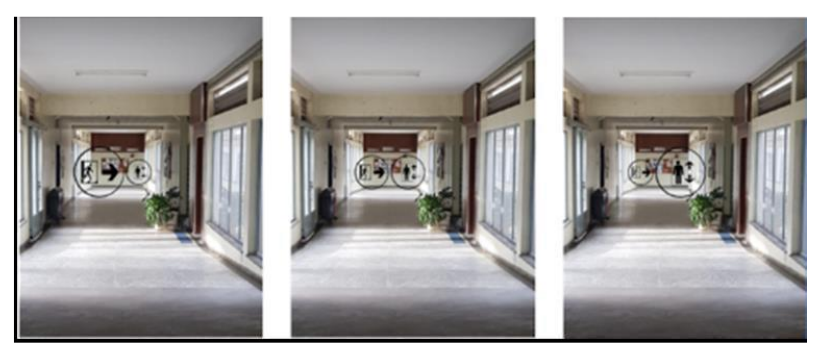

Figure 2. Environment with high contrast used in the experiment.

It was possible to observe that scenarios with low contrast between the environment and symbols are determinant for distance estimation, even if the symbols have sizes with a big difference. When symbols have the same size, it was possible to notice a great number of problems in the identification (59\% of participants). The environment with medium contrast presented better results regarding the comparison of symbols with bigger difference in size. Thus, in this environment the rate of errors $(35 \%)$ was higher when symbols were in the shadows area. On the other hand, the environments with high contrast have the best results in the distance estimative in all situations. However, it was noticed that the participants were able to identify the relative position between the symbols. Therefore, it was not considered any kind of metrics related to the position of the symbols.

The experiment showed that symbols designed using Gestalt Laws can inform about the distance and depth in navigation systems used in egocentric view. The results also indicate the participants did not face difficulties to estimate the difference in distance between the symbols. There is evidence that the evaluation of the symbol size can be replaced by the spatial knowledge related to the distance between the symbols and the user.

Besides that, users did not face difficulties in the symbol identification when the environment had high contrast. However, this is not true when considering the low contrast and low lighting. This indicates that a navigation system should consider the symbol design together with aspects of lightning, because it can step in the shape recognition process. Finally, it is recommended to consider the suitability of the $2 \mathrm{D}$ symbols to the AR system, regarding the aspects of dimension, transparency and size. 